\title{
SPOCKI promotes tumor growth and metastasis in human prostate cancer
}

This article was published in the following Dove Press journal:

Drug Design, Development and Therapy

18 July 2016

Number of times this article has been viewed

\author{
Qi Chen ${ }^{1, *}$ \\ Yuan-ting $\mathrm{Yao}^{2, *}$ \\ Huan $\mathrm{Xu}{ }^{\prime}$ \\ Yan-bo Chen' \\ Meng Gu' \\ Zhi-kang Cai' \\ Zhong Wang'
}

'Department of Urology, Shanghai 9th People's Hospital, Shanghai Jiao Tong University School of Medicine, 2Institute of Plant Physiology and Ecology, Chinese Academy of Sciences, Shanghai, People's Republic of China

*These authors contributed equally to this work
Correspondence: Zhong Wang;

Zhi-kang Cai

Department of Urology, Shanghai 9th People's Hospital, Shanghai Jiao Tong University School of Medicine, No 639

Zhizaoju Road, Shanghai 2000II,

People's Republic of China

Tel +862 2I 2327 I699

$\mathrm{Fax}+862163136856$

Email zhongwang2010@sina.com;

|33|1630565@|89.cn

\begin{abstract}
Prostate cancer is the most diagnosed noncutaneous cancer and ranks as the second leading cause of cancer-related deaths in American males. Metastasis is the primary cause of prostate cancer mortality. Survival rate is only $28 \%$ for metastatic patients, but is nearly $100 \%$ for patients with localized prostate cancers. Molecular mechanisms that underlie this malignancy remain obscure, and this study investigated the role of SPARC/osteonectin, cwcv, and kazallike domain proteoglycan 1 (SPOCK1) in prostate cancer progression. Initially, we found that SPOCK1 expression was significantly higher in prostate cancer tissues relative to noncancerous tissues. In particular, SPOCK1 expression was also markedly high in metastatic tissues compared with nonmetastatic cancerous tissues. SPOCK1 expression knockdown by specific short hairpin RNA in PC3 cells was significantly inhibited, whereas SPOCK1 overexpression in RWPE-1 cells promoted cell viability, colony formation in vitro, and tumor growth in vivo. Moreover, the SPOCK1 knockdown in PC3 cells was associated with cell cycle arrest in $\mathrm{G}_{0} / \mathrm{G}_{1}$ phase, while the SPOCK1 overexpression in RWPE-1 cells induced cell cycle arrest in S phase. The SPOCK1 knockdown in PC3 cells even increased cell apoptosis. SPOCK1 modulation was also observed to affect cancerous cell proliferation and apoptotic processes in the mouse model of prostate cancer. Additionally, the SPOCK1 knockdown decreased, whereas the SPOCK1 overexpression increased cell migration and invasion abilities in vitro. Injection of SPOCK1-depleted PC3 cells significantly decreased metastatic nodules in mouse lungs. These findings suggest that SPOCK1 is a critical mediator of tumor growth and metastasis in prostate cancer.
\end{abstract}

Keywords: SPOCK1, growth, metastasis, prostate cancer

\section{Introduction}

Prostate cancer is the most diagnosed noncutaneous cancer and ranks as the second leading cause of cancer-related deaths in American males. ${ }^{1}$ Based on recent statistics, 238,590 newly diagnosed cases of prostate cancer were reported; among these cases, 29,720 cases of American males were estimated to die in 2013, which makes this cancer the most serious health problem among male patients. ${ }^{2}$ Metastasis is the primary factor for prostate cancer mortality. ${ }^{3}$ The 5-year survival rate for patients diagnosed with metastatic prostate cancer is estimated to be $28 \%$; in contrast, this rate is nearly $100 \%$ for patients with localized tumors. ${ }^{4}$ The worse finding is that the overall survival has not changed in the last 20 years among patients who suffer from metastatic prostate cancer. However, an approximately $40 \%$ decrease in the mortality of this malignancy has been achieved over the last two decades. Hence, the means to prevent prostate cancer progression and to perform necessary interventions before this cancer metastasizes to other organs remain a major clinical challenge.

SPARC/osteonectin, cwcv, and kazal-like domain proteoglycan 1 (SPOCK1, also known as testican-1) is a proteoglycan that belongs to a novel $\mathrm{Ca}^{2+}$-binding $\mathrm{BY}$
hereby accept the Terms. Non-commercial uses of the work are permitted without any further permission from Dove Medical Press Limited, provided the work is properly attributed. For permission for commercial use of this work, please see paragraphs 4.2 and 5 of our Terms (https://www.dovepress.com/terms.php). 
proteoglycan family. Members of this family, which share a similar structure that includes N-terminus, follistatin-like domain, and C-terminus, are implicated in cell proliferation, cell-cell adhesion, and migration. ${ }^{5}$ SPOCK1 has been observed to play crucial roles in cell cycle regulation, cell apoptosis, DNA repair, and metastasis. ${ }^{6}$ SPOCK1 expression was fairly high in the brain. ${ }^{7}$ This proteoglycan is also present in other tissues, such as cartilages ${ }^{8}$ and myoblasts. ${ }^{9}$ More interestingly, a lot of evidence has shown that SPOCK1 plays critical roles in hepatocellular carcinoma progression ${ }^{10}$ and glioblastoma invasion. ${ }^{11}$ SPOCK1 can regulate the epithelial-mesenchymal transition (EMT) process in lung cancer. ${ }^{12}$ Moreover, SPOCK1-mediated EMT signaling confers acquired resistance to lapatinib in HER2-positive gastric cancer. ${ }^{13}$ SPOCK1 can even serve as a potential prognostic marker in gallbladder cancer. ${ }^{14}$ All these studies suggest the extensive roles of SPOCK1 in human tumorigenesis. The most noticeable finding is that SPOCK1 was first isolated in human testes, and eventually, two studies reported the aberrant expression of SPOCK1 in prostate cancer. ${ }^{15,16}$ However, minimal data have shown whether SPOCK1 plays any role in prostate tumorigenesis and prostate cancer progression.

This study aimed at investigating the SPOCK1 expression profiles in prostate cancer, with a special focus on its expression in metastatic tissues. For functional studies, specific short hairpin RNA (shRNA) against SPOCK1 (shSPOCK1) and its expression plasmid were employed. A systemic study of the SPOCK1 modulation effects on tumor growth and metastasis in prostate cancer will be conducted in this study.

\section{Materials and methods Human samples and immunohistochemistry analysis}

Fifty cases of prostate cancer patients who were admitted to Shanghai 9th People's Hospital were acquired. None of these patients received preoperative chemotherapy, radiotherapy, or androgen therapy. The tumor tissues from each patient as well as the matched adjacent nontumor tissue were obtained with the patient's authorization. All experiments included in this research were in compliance with the defined policies and protocols. All patients provided written consent for the use of their tumor tissue for clinical research, and the JiaoTong University Shanghai Cancer Center Ethical Committee approved the research protocol. For immunohistochemistry (IHC) analysis, each sample was fixed in 4\% buffered paraformaldehyde and embedded in paraffin. Samples were cut into $4 \mu \mathrm{m}$ sections. The deparaffinized sections were then boiled in a microwave in 0.1 $\mathrm{M}$ citric acid buffer ( $\mathrm{pH}$ 6.0). Subsequently, these sections were incubated overnight with corresponding primary antibodies at $4^{\circ} \mathrm{C}$. Secondary antibody was then incubated with the slides at room temperature for 1 hour. Immunoreactivity was developed in $0.05 \%$ diaminobenzidine that contained $0.01 \% \mathrm{H}_{2} \mathrm{O}_{2}$. Each slide was graded as $1-6$ based on the staining density and positive staining rate by an experienced pathologist.

\section{Mouse xenograft model}

Twenty-four, 6-week-old male nude mice were commercially purchased from SLRC Laboratory Animal Co. (Shanghai, People's Republic of China) and were evenly divided into four groups ( $n=6$ for each group). Approximately $2 \times 10^{5}$ of prostate cancer cells PC3 with or without SPOCK1 knockdown or RWPE-1 cells with or without SPOCK1 overexpression were suspended in phosphate-buffered saline (PBS) and were subcutaneously injected into each mouse group. All mice were kept in a light- and temperature-controlled environment, with access to water and food ad libitum. In the 4-week observation period after injection, the volume of each tumor was measured twice a week. All efforts were made to minimize the sufferings of the mice.

All animal experiments were performed in compliance with the Guidelines for the Care and Use of Laboratory Animals of Shanghai JiaoTong University, and the protocol was approved by the Committee on the Ethics of Animal Experiments of Shanghai JiaoTong University.

\section{Cell culture and transfection}

The nonmalignant prostate cell line RWPE-1 and four human prostate cancer cell lines, namely, 22Rv1, PC3, DU145, and LNCaP, were purchased from American Type Culture Collection (ATCC, Manassas, VA, USA). Cells were cultured in recommended medium supplied with $10 \%$ fetal bovine serum (Gibco, Carlsbad, CA, USA) and maintained in a humidified incubator with $5 \% \mathrm{CO}_{2}$ at $37^{\circ} \mathrm{C}$. Notably, PC3 cells and RWPE-1 cells were transfected with specific shRNA against SPOCK1 and SPOCK1 plasmid, respectively, with the transfection reagent Lipofectamine 2000 (Invitrogen, Carlsbad, CA, USA).

\section{Total RNA extraction and real-time PCR}

Total RNA from both clinical tissues and cell lines were extracted with Trizol Reagent (TaKaRa, Shiga, Japan) in accordance with the manufacturer's instructions. Quality and concentration of RNAs were determined using Nanodrop 2000 (Thermo Scientific, Pittsburgh, PA, USA). First-strand cDNAs were generated with the PrimeScript RT Master Mix Perfect Real Time Kit (TaKaRa). All real-time PCR (RT-PCR) were operated with the SYBR 
Premix Ex Taq Kit (TaKaRa) using an ABI PRISM 7900 Real-time System. GAPDH was included as an internal control, and the primers used were shown as follows: SPOCK1: 5'-CCCGTTACTGCCGGTGATTA-3' (forward) and 5'-CCAGGTCTGGACAAGCTGAG-3' (reverse); GAPDH: 5'-GTGGACATCCGCAAAGAC-3' (forward) and 5'-AAAGGGTGTAACGCAACTA-3' (reverse).

\section{Western blot analysis and antibodies}

Total protein lysates were obtained from cultured cells with lysis buffer supplemented with protease inhibitors. After determination of the qualities and concentrations of each extraction with BCA protein assay kit (Beyotime, Nanjing, People's Republic of China), $50 \mu \mathrm{g}$ whole proteins were loaded onto a $12 \%$ sodium dodecyl sulfate-polyacrylamide gel electrophoresis gel (Invitrogen). Afterward, proteins were transferred to nitrocellulose membrane, and nonspecific bindings were eliminated with $5 \%$ milk/TBST. Following overnight incubation with primary antibodies at $4^{\circ} \mathrm{C}$, the membranes were incubated with horseradish peroxidase-conjugated secondary antibodies for 1 hour at $37^{\circ} \mathrm{C}$. Immunoreactivity was detected with enhanced chemiluminescent autoradiography (Thermo Scientific). Primary antibodies against SPOCK1, Cdc25C, cyclin D1, cyclin B1, GAPDH, and secondary antibodies were commercially purchased from Santa Cruz Biotechnology (Santa Cruz, CA, USA). Primary antibodies against PI3K, AKT, Bad, Bcl-xL, Bcl-2, and antibodies for detection of phosphorylated PI3K (p-PI3K) at Y607 and phosphorylated AKT (p-AKT) at Ser473 were bought from Abcam (Cambridge, MA, USA). Matrix metalloproteinase 3 (MMP3) and MMP9 antibodies were purchased from Cell Signaling Technology (Danver, MA, USA).

\section{Cell proliferation assay}

Prostate cancer cells, PC3 and RWPE-1, were seeded into 96-well plates at a density of $3 \times 10^{3}$ cells per well, incubated overnight. After transfection with specific shSPOCK1 in PC3 cells and SPOCK1 plasmid into RWPE-1 cells, the cells were incubated for another 72 hours at $37^{\circ} \mathrm{C}$. Cell viabilities were detected in the subsequent 6 days with 3-(4,5-dimethylthiazol2-yl)-2,5-diphenyltetrazoliumbromide methyl thiazolyl tetrazolium (MTT) solution. Approximately $0.2 \mathrm{mg}$ MTT solution was appended into each well for the monitored days. After incubation for an additional 4 hours, the medium from each well was discarded, and $200 \mu \mathrm{L}$ dimethyl sulfoxide was added. The plate was shaken-cultured for 5 minutes, and optical density was examined at $570 \mathrm{~nm}$ absorption.

\section{Colony formation assay}

PC3 and RWPE-1 cells were transfected with shSPOCK1 and SPOCK1 expression plasmid, respectively, prior to test. The cells were seeded into 6-well plates at a density of 500 cells per well. After incubation for an additional 3 weeks, the plates were fixed with chilled methanol and stained with $0.1 \%$ crystal violet for 5 minutes. Visible colonies were manually calculated in triplicate.

\section{Cell cycle assay}

For cell cycle evaluation, scramble shRNA or shSPOCK1treated PC 3 cells and vector or SPOCK1 plasmid-transfected RWPE-1 cells were trypsinized and fixed in ice-cold methanol for 20 minutes. After RNase treatment, the cells were treated with protease inhibitor (PMSF, $50 \mu \mathrm{g} / \mathrm{mL}$ ) for 30 minutes, and the fluorescence intensities were detected using the FC500 flow cytometer (Beckman, Pasadena, CA, USA) with FL-1H filter. Furthermore, Modfit 2.0 software (Verity Software House Inc, Topsham, ME, USA) was used to quantify the cell numbers in $\mathrm{G}_{0} / \mathrm{G}_{1}, \mathrm{~S}$, and $\mathrm{G}_{2} / \mathrm{M}$ phases.

\section{Cell apoptosis detection}

The Annexin V/propidium iodide (PI) assay was performed in accordance with the manufacturer's recommendation (Invitrogen). Briefly, $10^{5}$ cells were plated into 6-well plates (Corning Inc, Corning, NY, USA) and incubated for 48 hours. Subsequently, the cells were collected and washed with precold PBS. Later, the cells were centrifuged and resuspended in $100 \mu \mathrm{L}$ of binding buffer that contains $2.5 \mu \mathrm{L}$ fluorescein isothiocyanate-conjugated Annexin- $\mathrm{V}$ and $1 \mu \mathrm{L}$ PI $(100 \mu \mathrm{g} / \mathrm{mL})$, which was followed by further incubation at room temperature for 15 minutes in a dark environment. At least 10,000 events were collected and analyzed through flow cytometry (BD Biosciences, San Diego, CA, USA).

Cell apoptosis was also assessed by Hoechst 33342 staining. Briefly, PC3 cells were washed with PBS after fixation with methanol:acetic acid $(3: 1, \mathrm{v} / \mathrm{v})$ and stained with Hoechst 33342 stain $(5 \mu \mathrm{g} / \mathrm{mL})$ for 10 minutes. Morphological changes in the cell nuclei were observed under a fluorescence microscope (Leica, Wetzlar, Germany).

\section{Transwell assay}

Cells were seeded in 24-well plates and transfected with specific SPOCK1 shRNA or plasmid. Afterward, cells were harvested in serum-free media as single cell suspension and approximately $3 \times 10^{4}$ cells were inoculated in the upper chamber (Sigma, St Louis, MO, USA). Meanwhile, the lower 
chamber was filled with $600 \mu \mathrm{L}$ corresponding medium supplemented with $10 \%$ fetal bovine serum. For the invasion assay, the chamber was coated with Matrigel (Trevigen, Inc, Gaithersburg, MD, USA) at the buffer 6 hours ahead of time at $37^{\circ} \mathrm{C}$. After incubation for another 7 hours (migration) or 9 hours (invasion), cells were fixed with precold methanol for 15 minutes and stained with $0.1 \%$ crystal violet for 5 minutes. The images were acquired at a $200 \times$ magnification. This analysis was repeated at least three times with each time experiment performed in duplicate.

\section{Statistical analysis}

All statistical analyses were determined using Prism 5 (GraphPad Software, La Jolla, CA, USA). Comparison between groups was presented using unpaired $t$-tests. $P<0.05$ were considered to be significant. The results were exhibited as means \pm standard deviation.

A

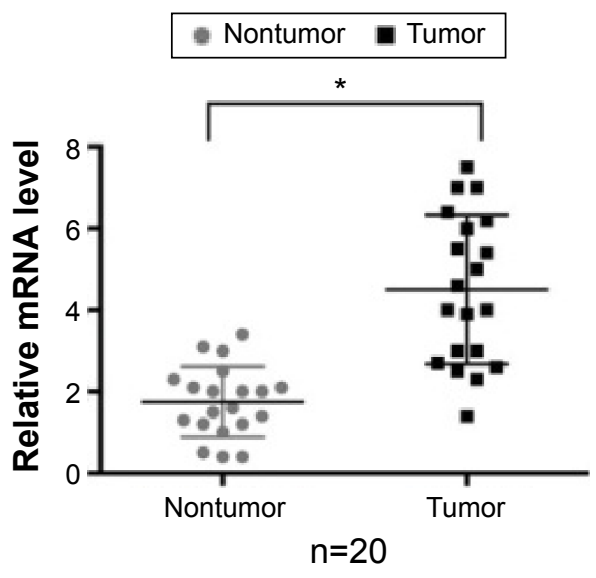

C

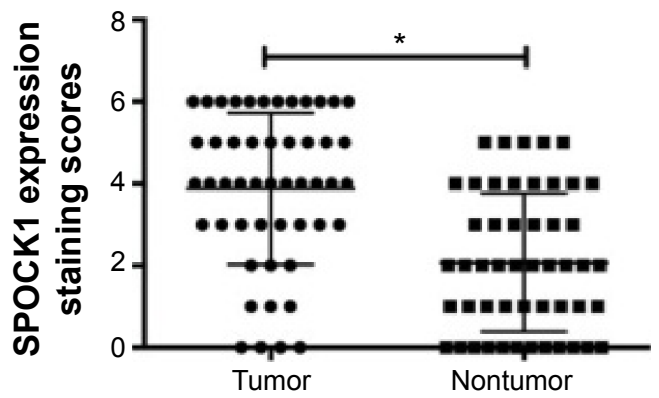

\section{Results}

SPOCKI is overexpressed in prostate cancer tissues

Initially, we performed qRT-PCR analysis of the SPOCK1 mRNA levels in 20 consecutive prostate cancer cases. Our data showed that the relative mean mRNA level of SPOCK1 in the cancerous tissues was approximately twofold of that in the adjacent noncancerous tissues (Figure 1A). Moreover, we performed IHC analysis in 50 prostate cancer cases. The IHC staining revealed that SPOCK1 was densely stained in the tumor tissues, whereas this proteoglycan was rarely detected in nontumor tissues (Figure 1B). Further analysis showed that 32 of the 50 cases $(64 \%)$ were strongly stained with a score over 4 in the tumor samples. On the contrary, only 13 of the 50 cases $(26 \%)$ were strongly stained with a score over 4 in the nontumor samples (Figure 1C). Furthermore, the average

B

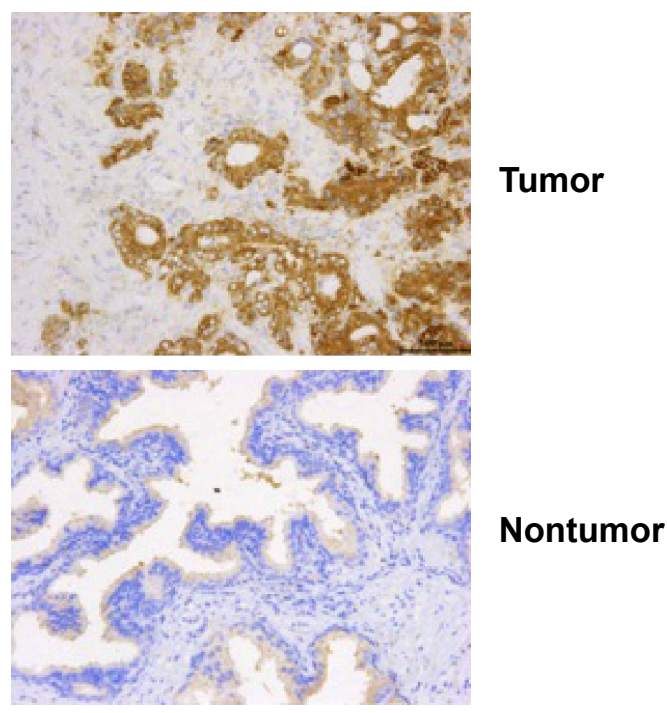

D

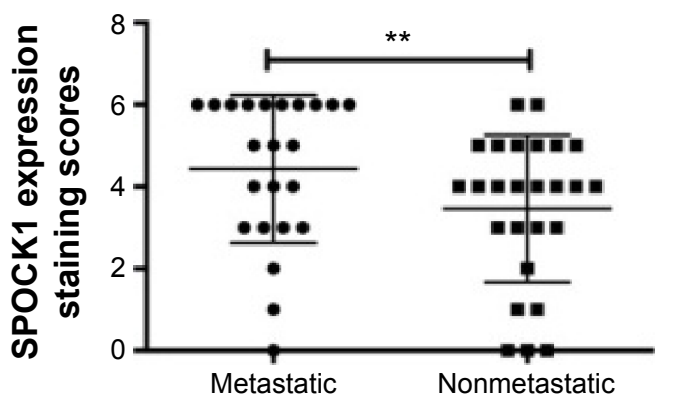

Figure I SPOCKI is overexpressed in prostate cancer tissues.

Notes: (A) qRT-PCR analysis of SPOCKI in 20 cases of prostate cancer. Levels of SPOCKI in tumor and the adjacent nontumor tissues were detected and compared. (B) IHC analysis of the protein expression of SPOCKI in 50 cases of prostate cancer. Representative images showing the high staining of SPOCKI in tumor tissues were shown. (C) After the scoring of IHC staining, all the 50 tumor cases and 50 nontumor cases were classified into each score. Staining scores of SPOCKI in the tumor tissues were significantly higher than the nontumor tissues. (D) The 50 cases were divided into those with metastasis $(n=24)$ and those without $(n=26)$. It was further shown by IHC that the average staining score of SPOCKI in metastatic tissues was significantly higher than the nonmetastatic tissues. $* P<0.001$; $* * P<0.05$, as indicated.

Abbreviations: SPOCKI, SPARC/osteonectin, cwcv, and kazal-like domain proteoglycan I; IHC, immunohistochemistry analysis; qRT-PCR, quantitative real-time polymerase chain reaction. 
staining score of SPOCK1 in the 24 metastatic cases was significantly higher than that of the 26 nonmetastatic cases (Figure 1D). These observations strongly suggest the high SPOCK1 expression in prostate cancer tissues, particularly in the metastatic tissues.

\section{Successful modulation of SPOCK I expression in prostate cancer cells}

Furthermore, we performed Western blot analysis of SPOCK1 in five prostate cancer cell lines. Our data showed that SPOCK1 in these cell lines was differentially expressed, with its highest expression present in PC3 cells and least level of expression in RWPE-1 cells (Figure 2A). This result made PC3 and RWPE-1 our optimal cell lines for subsequent functional analyses. We employed specific shRNA to deplete SPOCK1 expression in the PC3 cell line and to upregulate SPOCK1 in the RWPE-1 cell line with its expression plasmid. Transfection of PC3 cells with the specific shSPOCK1 significantly decreased the SPOCK1 mRNA level in PC3 cells (Figure 2B), whereas SPOCK1 plasmid transfection into RWPE-1 cells increased its mRNA level by up to 4.5 -fold (Figure 2C). Consistently, the SPOCK1 protein level was decreased in response to its specific shRNA and increased through transfection of its expression plasmid (Figure 2D). These data confirmed the successful construction of prostate cancer cells that were stably depleted of SPOCK1 (PC3 cells) or overexpressed SPOCK1 (RWPE-1 cells).

\section{Modulation of SPOCKI expression affected cell proliferation in vitro}

To study the effects of SPOCK 1 modulation on prostate cancer cell proliferation, we performed MTT assay to assess cell viability in PC3 cells (Figure 3A) and RWPE-1 cells (Figure 3B). Cell numbers were monitored for 6 consecutive days in both cell lines. In PC3 cells, SPOCK1 knockdown was observed to decrease the cell viability since day 3 . By day 6 , cell viability was only half that of the control cells (Figure 3A). On the contrary, SPOCK1 overexpression in RWPE-1 cells increased cell viability since day 3 (Figure 3B). Moreover, we performed a colony formation assay (Figure 3C). SPOCK1 knockdown was shown to significantly decrease colony formation in PC3 cells, whereas SPOCK1 overexpression markedly increased the number of colonies in RWPE-1 cells (Figure 3D).

\section{Modulation of SPOCKI interrupted cell cycle progression and cell apoptosis process}

Cell cycle progression was subsequently assessed through flow cytometry (Figure 4A). Our results showed that in PC3 cells, when SPOCK1 was depleted, cell percentage in $\mathrm{G}_{0} / \mathrm{G}_{1}$
A

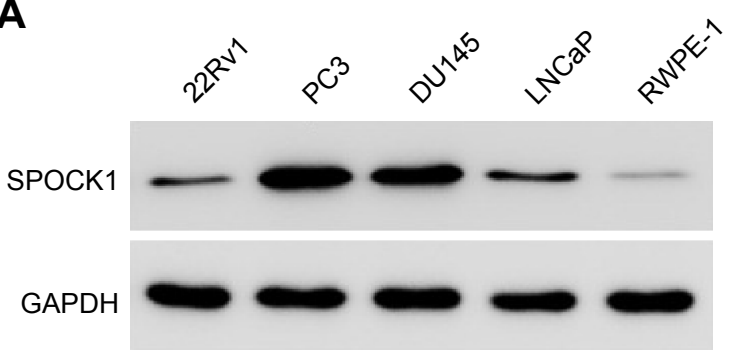

C

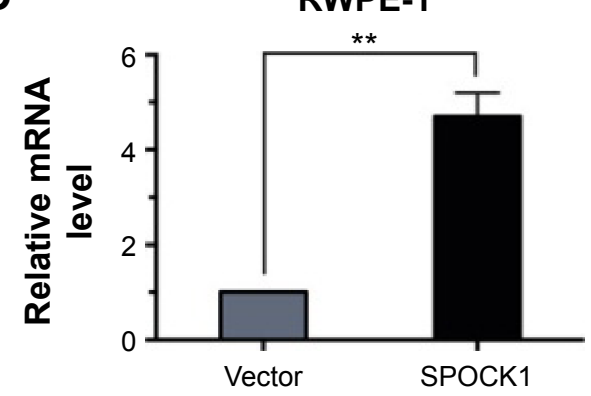

B

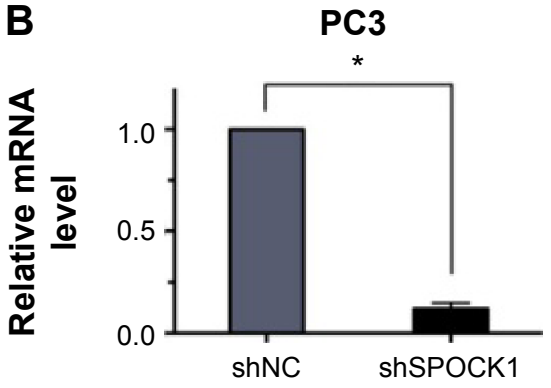

D

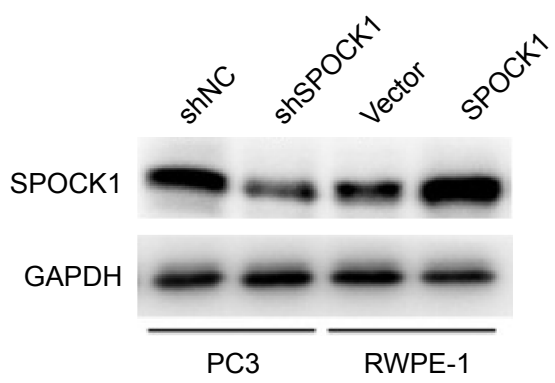

Figure 2 Successful modulation of SPOCKI expression in prostate cancer cells.

Notes: (A) Immunoblot analysis of the protein level of SPOCKI in 5 prostate cancer cell lines. The protein level of SPOCKI was highest in PC3 cells, while it was least expressed in RWPE-I cells. (B, C) Transfection of specific shSPOCKI decreased the mRNA level of SPOCKI in PC3 cells (B), while transfection of its expression plasmid increased its mRNA level in RWPE-I cells (C). (D) Immunoblot analysis further confirmed that the protein level of SPOCKI was decreased by transfection of shSPOCKI and increased by transfection of SPOCKI plasmid. $* P<0.000$ I; $* * P<0.01$.

Abbreviations: SPOCKI, SPARClosteonectin, cwcv, and kazal-like domain proteoglycan I; shRNA, short hairpin RNA; shSPOCKI, shRNA against SPOCKI; GAPD, reduced glyceraldehyde-phosphate dehydrogenase; shNC, negative control. 


\section{A}

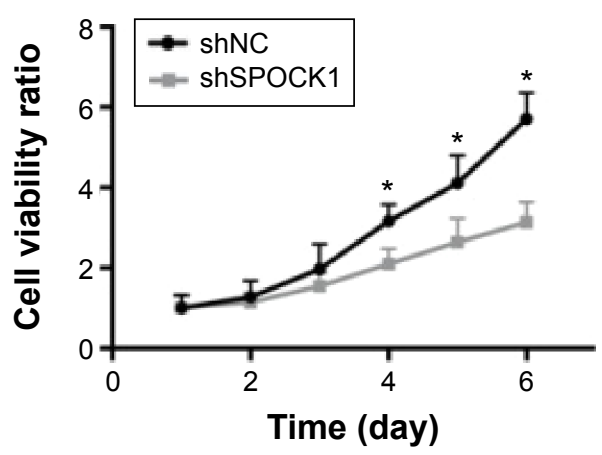

C

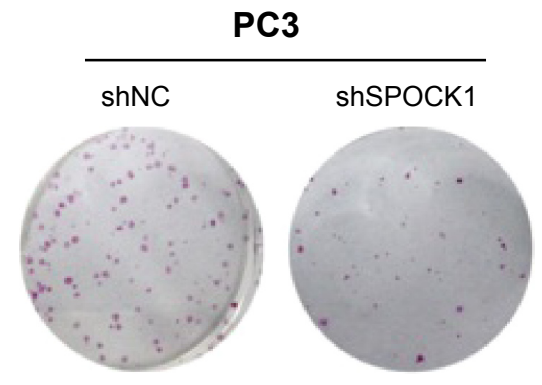

D

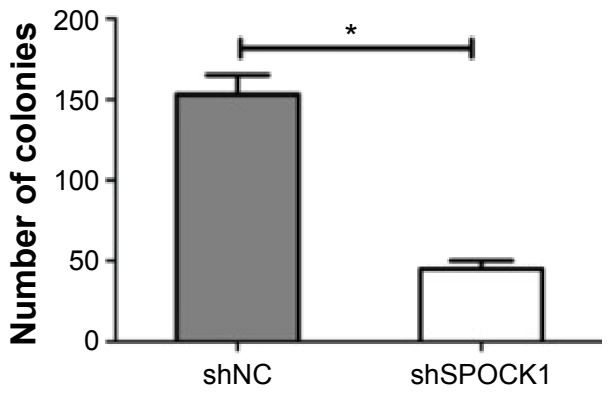

B

RWPE-1

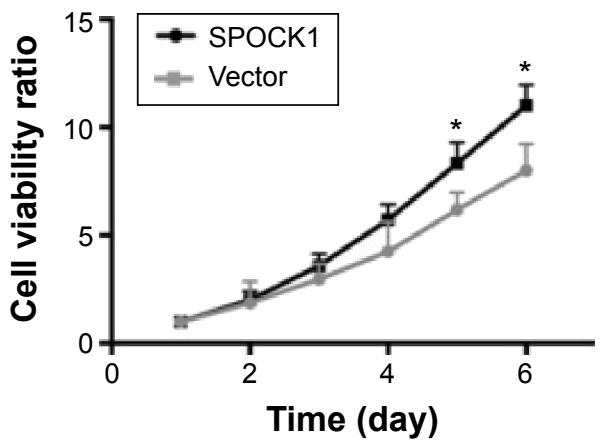

RWPE-1
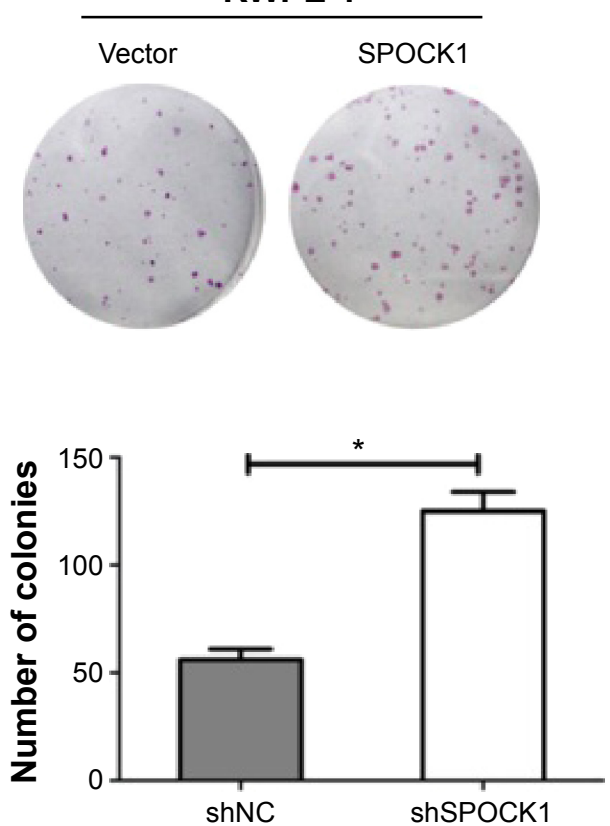

Figure 3 Modulation of SPOCKI expression affected cell proliferation in vitro.

Notes: Effects of SPOCKI knockdown in PC3 cells (A) and overexpression in RWPE-I cells (B) on cell viability on a consecutive of 6 days. Colony formation ability was assessed after modulation of SPOCKI in prostate cancer cells. Colony was stained with crystal violet (C). Quantification of the colonies showed that knockdown of SPOCKI in PC3 cells (left graph) significantly decreased, whereas upregulation of SPOCKI in RWPE-I cells (right graph) increased the number of colonies (D). *P $<0.01$.

Abbreviations: SPOCKI, SPARC/osteonectin, cwcv, and kazal-like domain proteoglycan I; shSPOCKI, shRNA against SPOCKI; shNC, negative control.

phase was significantly increased from $40 \%$ to nearly $70 \%$, whereas cell percentage in $\mathrm{S}$ phase and $\mathrm{G}_{2} / \mathrm{M}$ phase was decreased accordingly. On the contrary, when SPOCK1 was upregulated in RWPE- 1 cells, the cell percentage in $\mathrm{G}_{0} / \mathrm{G}_{1}$ phase was decreased, which was associated with increased cell proportion in $\mathrm{S}_{\text {and }} \mathrm{G}_{2} / \mathrm{M}$ phases (Figure 4B). The critical regulators for cell cycle progression, such as $\mathrm{Cdc} 25 \mathrm{C}$, cyclin $\mathrm{B} 1$, and cyclin D1, were all consistently altered in response to SPOCK1 expression (Figure 4C); this outcome confirmed the notion of SPOCK1-mediated regulation of cell cycle progression. More interestingly, we assessed the cell apoptosis in PC3 cells with or without SPOCK1 knockdown. We found that when SPOCK1 was depleted, cell apoptosis was significantly promoted as compared with control PC3 cells (Figure 4D). Similarly, SPOCK1-depleted PC3 cells exhibited more severe nuclear fragmentation and chromatin condensation, which represented the apoptotic process. Apoptotic cell quantification revealed that shSPOCK1-treated PC3 cells were remarkably apoptotic, with the cell apoptosis rate as high as $8 \%$ (Figure 4E). These data suggest that SPOCK1 modulation interrupted cell cycle progression and affected cell survival.

\section{SPOCKI affected tumor growth in a mouse model}

To test the effect of SPOCK1 modulation on tumor growth in vivo, we established a xenograft model of human prostate 


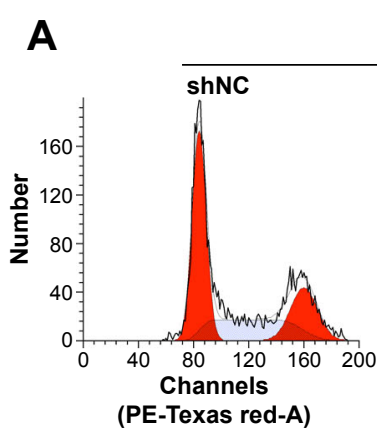

PC3
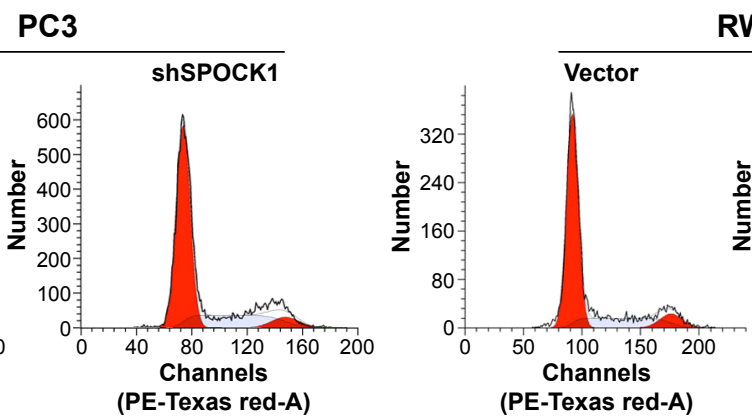

RWPE-1

B

PC3

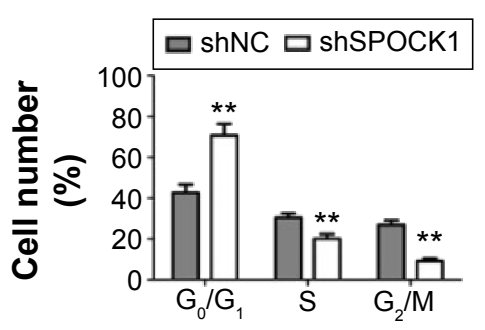

RWPE-1

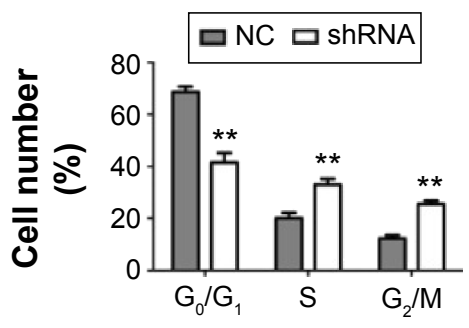

D

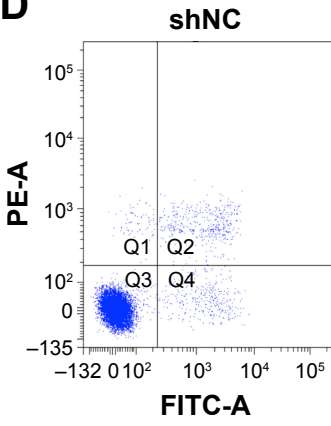

E

shNC

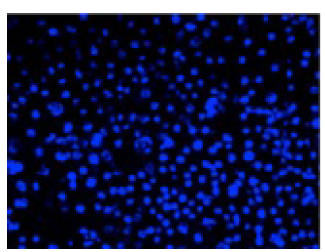

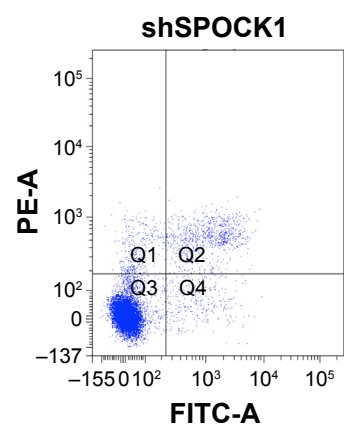

shSPOCK1

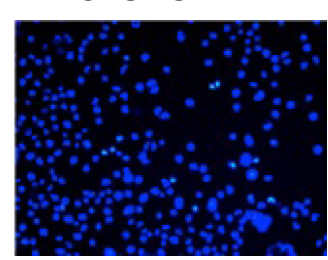

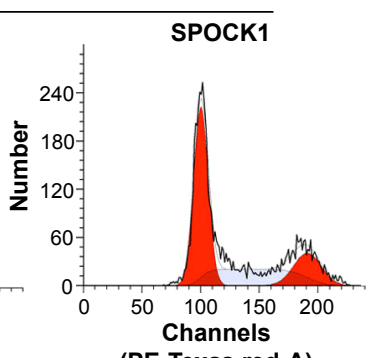

C
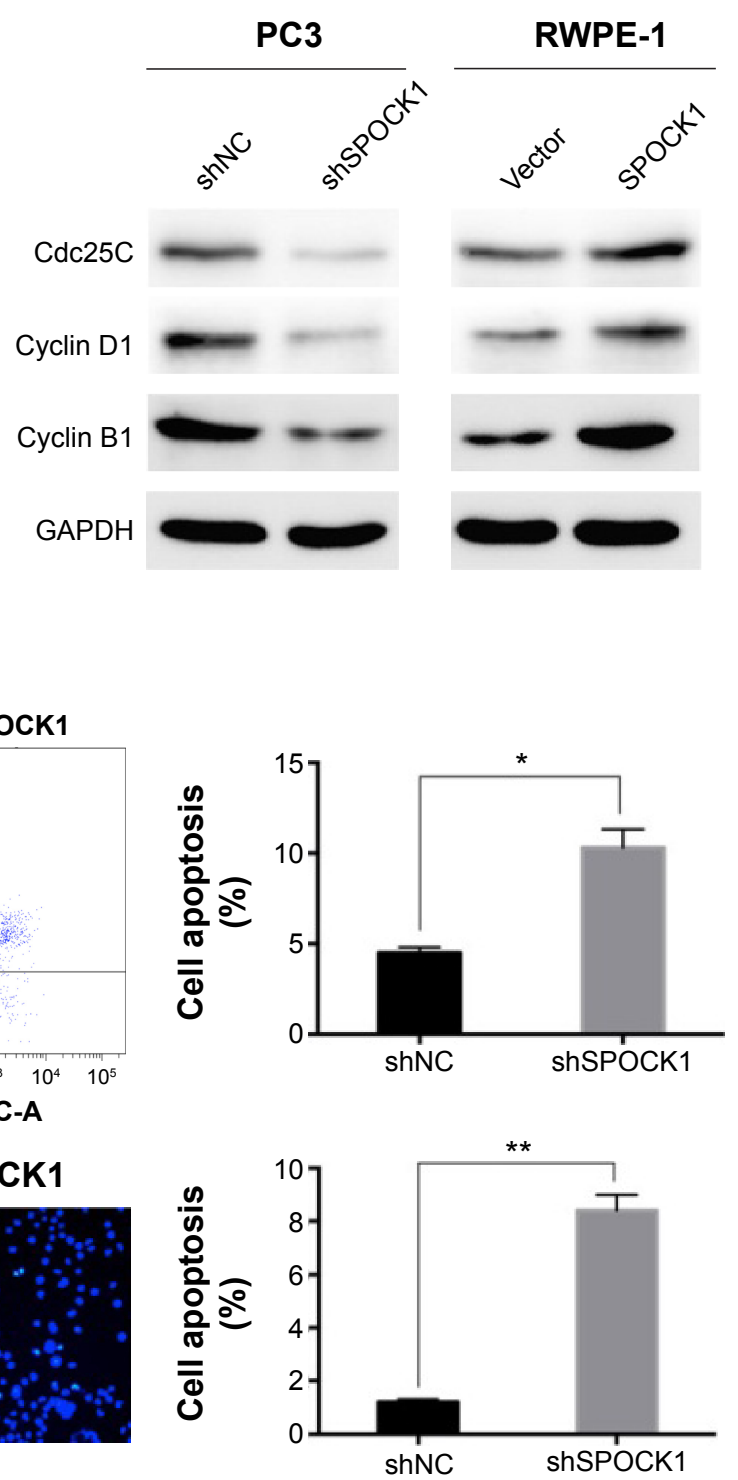

Figure 4 Modulation of SPOCKI interrupted cell cycle progression and cell apoptosis process.

Notes: (A, B) Cell cycle assessment showed that knockdown of SPOCKI in PC3 cells induced cell accumulation in $G_{0} / G_{1}$ phase. Overexpression of SPOCKI in RWPE-I cells decreased the cell proportion in $G_{0} / G_{1}$ phase, but increased cell percentage in $S$ phase and $G_{2} / M$ phase. (C) Immunoblot analysis of the critical cell cycle regulators. In SPOCKI-depleted PC3 cells, Cdc25C, cyclin BI, and cyclin DI were consistently decreased. However, in SPOCKI-overexpressed RWPE-I cells, expression of Cdc25C, cyclin BI, and cyclin DI were increased. (D) Annexin-PI analysis of cell apoptosis in PC3 with or without SPOCKI depletion. When SPOCKI was depleted, cell apoptosis rate was increased to 10\%, while it was less than $5 \%$ in the control PC3 cells. (E) Detection of morphological apoptosis with Hoechst 33342 staining. SPOCKI-depleted PC3 cells exhibited more severe nuclear fragmentation and chromatin condensation. The apoptosis rate was significantly higher than the control cells $(8 \%$ vs $1 \%)$. $* P<0.01$; $* * P<0.0001$.

Abbreviations: SPOCKI, SPARC/osteonectin, cwcv, and kazal-like domain proteoglycan I; shRNA, short hairpin RNA; shSPOCKI, shRNA against SPOCKI; shNC, negative control; GAPDH, reduced glyceraldehyde-phosphate dehydrogenase. 
cancer. Tumors were all dissected at the 4th week. Tumor size was shown to be visually smaller in PC3-depleted mouse group. On the contrary, tumor sizes in SPOCK1overexpressed group were markedly greater than those in the vector-injected control mouse group (Figure 5A). Periodic monitoring of tumor volume also showed that SPOCK1 depletion significantly slowed down tumor growth since the 2 nd week. By the 4 th week, tumor volume in shSPOCK 1 group was only approximately $30 \%$ of the shNC group (Figure 5B). The reverse effect was observed in SPOCK1-overexpressed RWPE-1 cells (Figure 5C). The effects of growth promotion by SPOCK1 overexpression was also confirmed by the IHC staining of PCNA, which is a cell proliferation marker. With the use of the mouse tumor samples, we performed histological and IHC analysis. IHC staining of PCNA revealed that this proliferation marker was markedly absent in SPOCK1-depleted tumor tissues, whereas this marker was strongly stained in SPOCK1-overexpressed tumor tissues. Expression of cleaved-caspase-3, which is a cell apoptosis marker, went the opposite way as compared with PCNA (Figure 5D). This outcome supported the findings that proliferation was inhibited and apoptosis was promoted by SPOCK1 depletion. Furthermore, immunoblot analysis of other apoptosis-related proteins, which include Bad, Bcl-xL, and Bcl-2, showed that the proapoptotic factor Bad was negatively regulated by SPOCK1, whereas antiapoptotic factors, Bcl-xL and Bcl-2, were positively regulated by SPOCK1 in both PC3 cells and RWPE-1 cells. AKT (p-AKT) and PI3K (p-PI3K) phosphorylation represent two critical pathways that phosphorylate Bad and led to its inactivation. ${ }^{17,18} \mathrm{We}$ also found that $\mathrm{p}-\mathrm{PI} 3 \mathrm{~K}$ and $\mathrm{p}-\mathrm{AKT}$ were positively regulated by SPOCK1 (Figure 5E). All these data strongly suggest that SPOCK1 promoted tumor growth and inhibited cell apoptosis in vivo.

\section{SPOCKI promoted metastasis in prostate cancer}

Afterward, we assessed whether SPOCK1 controlled the metastasis process in prostate cancer. Transwell assay showed that SPOCK1-depleted PC3 cells exhibited remark-
A

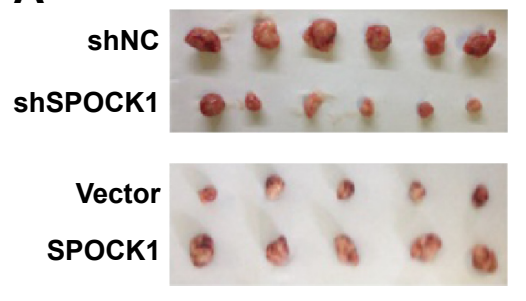

D

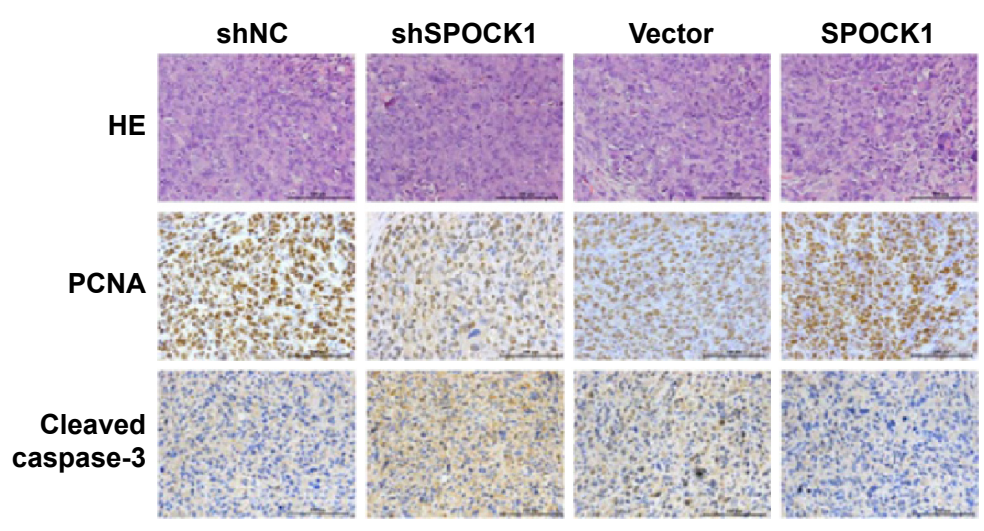

B
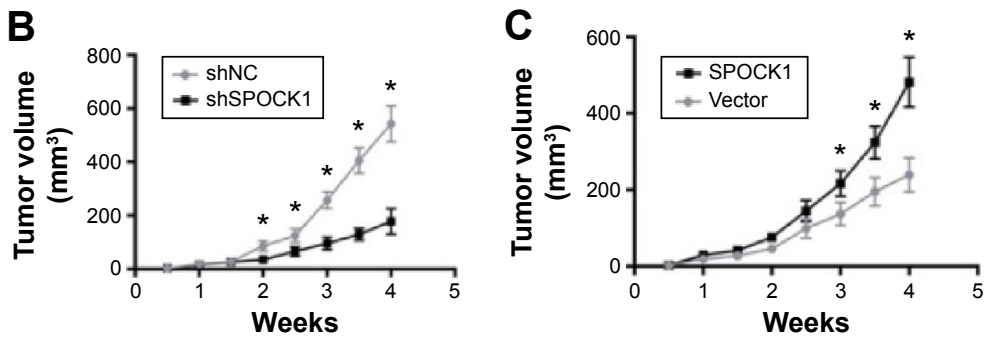

E

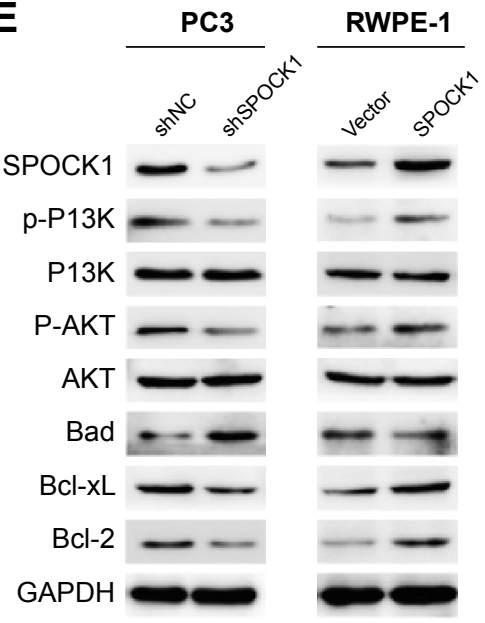

Figure 5 SPOCKI affected tumor growth in a mouse model.

Notes: (A) Tumor dissection showed that knockdown of SPOCKI caused tumor size smaller, while overexpression of SPOCKI enlarged tumor sizes. (B, C) periodic monitoring of tumor volume in PC3 cells and RWPE-I cells in a consecutive of 4 weeks. (D) Histology and immunohistochemistry analysis of the sections from the mouse model. PCNA, a proliferation marker, and cleaved-caspase-3 were detected for indicating cell proliferation and apoptosis, respectively. (E) Immunoblot analysis of expression of SPOCKI and a series of apoptosis-related proteins. It was observed that SPOCKI positively regulated antiapoptotic factors Bcl-2 and Bcl-xL as well as phosphorylation kinases of Bad such as P-PI3K and P-AKT. The proapoptotic factor Bad was negatively regulated by SPOCKI in both PC3 cells and RWPE-I cells. *P $<0.0 \mathrm{I}$.

Abbreviations: SPOCKI, SPARC/osteonectin, cwcv, and kazal-like domain proteoglycan I; PCNA, proliferating cell nuclear antigen; IHC, immunohistochemistry; p-PI3K, phosphorylated PI3K; p-AKT, phosphorylated AKT; HE, hematein eosin; shSPOCKI, shRNA against SPOCKI; shNC, negative control; GAPDH, reduced glyceraldehydephosphate dehydrogenase. 


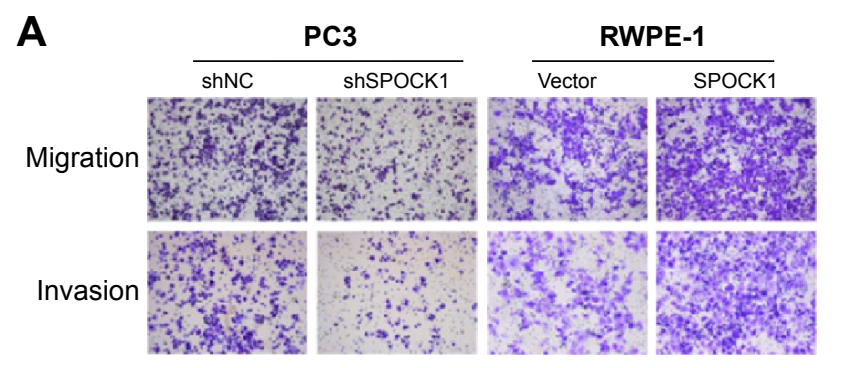

C
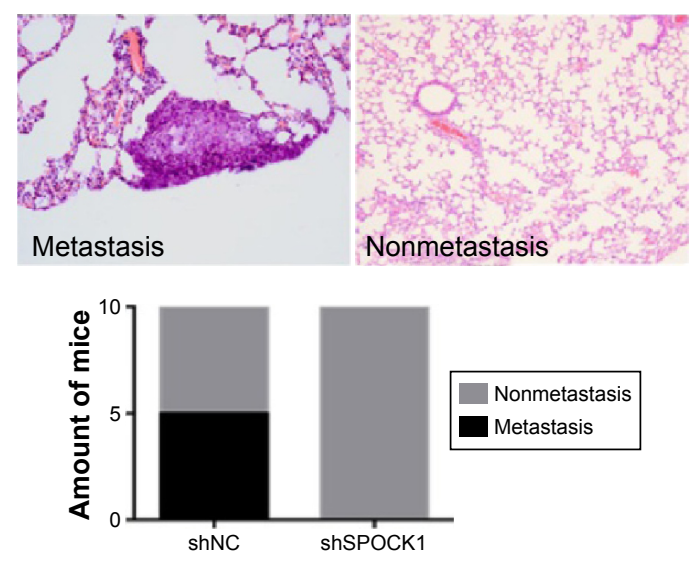

B
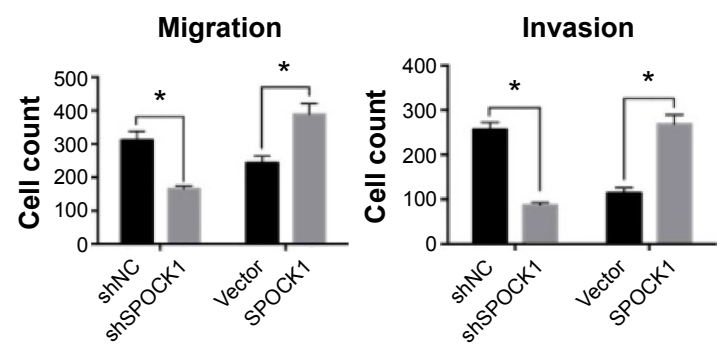

D

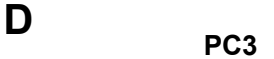

RWPE-1

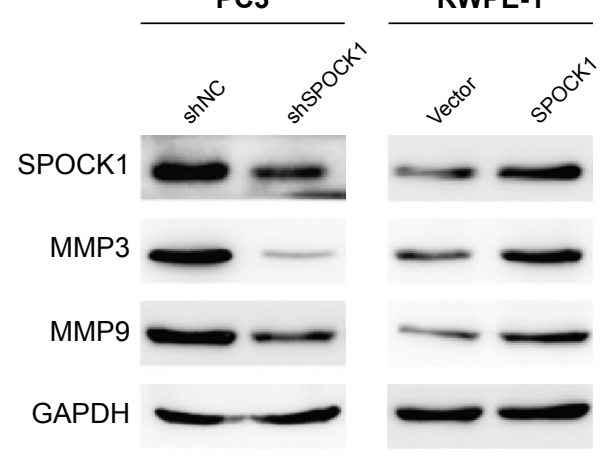

Figure 6 SPOCKI promoted metastasis in prostate cancer.

Notes: (A) Transwell assay showed that SPOCKI-depleted PC3 cells exhibited remarkably decreased migration and invasion abilities; whereas overexpression of SPOCK I in RWPE-I cells caused highly active migration and invasion. (B) Quantification of the transmigrated cells in the Transwell assay. $* P<0.01$. (C) Injection of PC 3 cells into two groups of mice ( $\mathrm{n}=10$ for each group) through caudal vein. PC3 cells were pretransfected with shSPOCKI or without shSPOCKI. It was observed that no mice in SPOCKI-depleted group exhibited lung nodules. (D) Immunoblot analysis of MMPs. The MMP3 and MMP9 were both positively regulated by SPOCKI in PC3 cells and RWPE-I cells.

Abbreviations: SPOCKI, SPARC/osteonectin, cwcv, and kazal-like domain proteoglycan I; MMPs, matrix metalloproteinases; shSPOCKI, shRNA against SPOCKI; shNC, negative control; GAPDH, reduced glyceraldehyde-phosphate dehydrogenase.

ably decreased migration and invasion abilities. On the contrary, SPOCK1 overexpression in RWPE-1 cells caused increased active migration and invasion (Figure 6A). In fact, in the migration assay, nearly half of the PC 3 cells were inhibited from migration when SPOCK1 was depleted; whereas a $60 \%$ increase in migration ability was observed for RWPE-1 cells. Likewise, nearly $70 \%$ of the PC3 cells were inhibited from invasion after SPOCK1 knockdown; whereas a $180 \%$ increase in invasion ability was achieved through SPOCK1 overexpression in RWPE-1 cells (Figure 6B). Furthermore, we injected an equal amount of PC3 cells with (shSPOCK1 group) or without shSPOCK1 (shNC group) into mice through the caudal vein ( $\mathrm{n}=10$ for each group). Our results showed that in the shNC group, five mice exhibited lung nodules (50\% metastasis rate), whereas none of the mice in shSPOCK1 group exhibited nodules in the lung (Figure 6C). These findings led to our conclusion that SPOCK1 promoted metastasis both in vitro and in vivo. Additionally, we also detected expression of MMPs, which are critical for cancer cell metastasis. MMP3 and MMP9 were both downregulated consistently by SPOCK1 knockdown and were both upregulated consistently by SPOCK1 overexpression (Figure 6D). All these conclusive data suggest that SPOCK1 could promote prostate cancer cell metastasis.

\section{Discussion}

Prostate cancer is the most commonly diagnosed cancer among male patients in many countries and accounts for approximately one in six of male cancer mortality in 2009 (ie, 124 deaths per 100,000 males). Metastases are the cause of $90 \%$ of human cancer deaths. ${ }^{3}$ A number of studies have documented the association between extracellular matrix gene SPOCK 1 and cancer cell metastasis; ${ }^{10,13,14}$ these studies suggest the extensive role of SPOCK1 in human tumorigenesis.

This study investigated the critical roles of SPOCK1 in tumor growth and metastasis in prostate cancer. SPOCK1 expression was initially found to be fairly high in prostate cancer tissues as compared with noncancerous tissues. In particular, SPOCK1 expression was higher in metastatic tissues relative to nonmetastatic ones. A previous study with microarray analysis has reported that SPOCK1 was upregulated or remained unchanged in prostate cancer. ${ }^{15}$ 
Another report stated that the SPOCK1 upregulation paralleled that of EPB41L4B, which is a cortical cytoskeleton protein that underlies the cell membrane. ${ }^{16}$ These data would implicate that SPOCK1 might be involved in cell-cell adhesion. Furthermore, our results showed that SPOCK1 knockdown in PC3 cells significantly slowed down cell proliferation, colony formation in vitro, and tumor growth in vivo; whereas SPOCK1 overexpression in RWPE-1 cells accelerated cell proliferation and colony formation as well as promoted tumor growth in the mouse model. The SPOCK1 knockdown in PC3 cells even arrested cell cycle progression in $\mathrm{G}_{0} / \mathrm{G}_{1}$ phase and induced significant cell apoptosis. Cyclin B1, cyclin D1, and Cdc25C are critical cell cycle regulators that promote checkpoint transitions during cell cycle progression. ${ }^{20-22}$ Cyclin B1, cyclin D1, and Cdc25C were all observed to be positively regulated by SPOCK1 in both PC3 cells and RWPE-1 cells. This finding reinforced the notion that SPOCK1 regulated cell cycle progression in prostate cancer.

Another interesting finding was that SPOCK1 promoted metastasis in prostate cancer. SPOCK1 is a glycoprotein that belongs to the extracellular matrix and is implicated in cell-cell adhesion. Metastasis requires stepwise processes that include specialized parameters of cell motility, such as adhesion, chemotaxis, and invasion. ${ }^{23}$ By employing two distinct approaches, ie, shRNA for knockdown and expression plasmid for upregulation, to modulate SPOCK1 expression, our study showed that SPOCK1 promoted cell migration and invasion in vitro. Moreover, SPOCK1 depletion in PC3 cells directly caused no lung nodules in the experimental mice. These results are conclusive that SPOCK1 mediates prostate cancer cell metastasis. In fact, as an extracellular matrix protein, SPOCK1 has been implicated in the metastasis of gallbladder cancer and hepatocellular carcinoma. ${ }^{10,14}$ The finding of SPOCK1 as a promoter for prostate cancer metastasis would suggest the extensive role of SPOCK1 in the malignant progression in human cancers.

However, the detailed mechanisms that underlie SPOCK1-mediated prostate cancer metastasis remain to be elucidated. One hypothesis would be that SPOCK1 regulated EMT process during cancer metastasis. The following four steps are required for EMT: 1) loss of tight junctions, adhesive junctions, and desmosomes; 2) cytoskeletal changes; 3) transcriptional shift; and 4) increased migration and motility. EMT interruption is widely recognized as an essential step for distal cancer metastasis. ${ }^{24}$ MMP3 and MMP9, for instance, are two mesenchymal markers that promote EMT and, hence, distal metastasis. ${ }^{25,26} \mathrm{We}$ observed that SPOCK1 positively regulated MMP3 and
MMP9 in both PC3 cells and RWPE-1 cells, respectively. This finding may be evidence that indicated the EMT regulation by SPOCK 1 in prostate cancer. Other supportive evidence included that SPOCK1 regulated the EMT process in lung cancer ${ }^{12}$ and that SPOCK1-mediated EMT signaling conferred acquired resistance to lapatinib in HER2-positive gastric cancer. ${ }^{13}$ Therefore, SPOCK1-regulated EMT signaling might explain why SPOCK1 promotes distal metastasis in prostate cancer. However, our hypothesis is still speculative and requires extensive functional studies for final validation.

SPOCK1 identification, as a key mediator of prostate cancer progression, is of great biological significance. Besides, SPOCK1 is also an AR-dependent gene and AR signaling continues to be active in almost all stages of prostate cancer. The targeting of SPOCK 1 may supplement the therapy with AR antagonist in prostate cancer. SPOCK1 was initially isolated from the testes. Our findings may suggest critical roles for SPOCK1 in disease development in the genital system. More importantly, SPOCK1 has always been implicated in human cancer progression. Our data may confirm that SPOCK1 exerts extensive oncogenic activities in human tumorigenesis.

In summary, we identified that SPOCK1 played critical roles in tumor growth and metastasis in prostate cancer. Although detailed mechanisms remain to be elucidated, the critical role of SPOCK1 in prostate cancer may provide evidence for development of novel therapeutics against SPOCK1 for the treatment and early detection of prostate cancer.

\section{Acknowledgments}

This study was supported by grants from the Shanghai Pudong New Area Health and Family Planning project (number PW 2013D-3), Key Disciplines Group Construction Project of Pudong Health Bureau of Shanghai (PWZxq2014-11), and Key Project of Science and Technology of Shanghai (number 134119a9800).

\section{Disclosure}

The authors report no conflicts of interest in this work.

\section{References}

1. Su B, Gao L, Baranowski C, Gillard B. A genome-wide RNAi screen identifies FOXO4 as a metastasis-suppressor through counteracting PI3K/AKT signal pathway in prostate cancer. PLoS One. 2014;9(7): e101411.

2. Siegel R, Naishadham D, Jemal A. Cancer statistics. 2013. CA Cancer J Clin. 2013;63(1):11-30.

3. Gupta GP, Massague J. Cancer metastasis: building a framework. Cell. 2006;127(4):679-695. 
4. Li S, Ma Y, Xie C, et al. EphA6 promotes angiogenesis and prostate cancer metastasis and is associated with human prostate cancer progression. Oncotarget. Epub May 27, 2015.

5. Ahel D, Horejsi Z, Wiechens N, et al. Poly(ADP-ribose)-dependent regulation of DNA repair by the chromatin remodeling enzyme ALC1. Science. 2009;325(5945):1240-1243.

6. Chen $\mathrm{L}, \mathrm{Hu} \mathrm{L}$, Chan $\mathrm{TH}$, et al. Chromodomain helicase/adenosine triphosphatase DNA binding protein 1-like (CHD11) gene suppresses the nucleus-to-mitochondria translocation of nur77 to sustain hepatocellular carcinoma cell survival. Hepatology. 2009;50(1):122-129.

7. Marr HS, Basalamah MA, Bouldin TW, et al. Distribution of testican expression in human brain. Cell Tissue Res. 2000;302(2):139-144.

8. Hausser HJ, Decking R, Brenner RE. Testican-1, an inhibitor of proMMP-2 activation, is expressed in cartilage. Osteoarthritis Cartilage. 2004;12(11):870-877.

9. Genini M, Schwalbe P, Scholl FA, Schafer BW. Isolation of genes differentially expressed in human primary myoblasts and embryonal rhabdomyosarcoma. Int J Cancer. 1996;66(4):571-577.

10. Li Y, Chen L, Chan TH, et al. SPOCK1 is regulated by CHD1L and blocks apoptosis and promotes HCC cell invasiveness and metastasis in mice. Gastroenterology. 2013;144(1):179-191.

11. Colin C, Baeza N, Bartoli C, et al. Identification of genes differentially expressed in glioblastoma versus pilocytic astrocytoma using Suppression Subtractive Hybridization. Oncogene. 2006;25(19):2818-2826.

12. Miao L, Wang Y, Xia H, et al. SPOCK1 is a novel transforming growth factor-beta target gene that regulates lung cancer cell epithelialmesenchymal transition. Biochem Biophys Res Commun. 2013;440(4): 792-797.

13. Kim HP, Han SW, Song SH, et al. Testican-1-mediated epithelialmesenchymal transition signaling confers acquired resistance to lapatinib in HER2-positive gastric cancer. Oncogene. 2014;33(25): 3334-3341.

14. Shu YJ, Weng H, Ye YY, et al. SPOCK1 as a potential cancer prognostic marker promotes the proliferation and metastasis of gallbladder cancer cells by activating the PI3K/AKT pathway. Mol Cancer. 2015; 14(1): 12 .
15. Wlazlinski A, Engers R, Hoffmann MJ, et al. Downregulation of several fibulin genes in prostate cancer. Prostate. 2007;67(16):1770-1780.

16. Schulz WA, Ingenwerth M, Djuidje CE, et al. Changes in cortical cytoskeletal and extracellular matrix gene expression in prostate cancer are related to oncogenic ERG deregulation. BMC Cancer. 2010; 10:505.

17. Datta SR, Dudek H, Tao X, et al. Akt phosphorylation of BAD couples survival signals to the cell-intrinsic death machinery. Cell. 1997;91(2): 231-241.

18. Zhao S, Konopleva M, Cabreira-Hansen M, et al. Inhibition of phosphatidylinositol 3-kinase dephosphorylates BAD and promotes apoptosis in myeloid leukemias. Leukemia. 2004;18(2):267-275.

19. Gronberg H. Prostate cancer epidemiology. Lancet. 2003;361(9360): 859-864.

20. Chou YW, Zhang L, Muniyan S, et al. Androgens upregulate Cdc25C protein by inhibiting its proteasomal and lysosomal degradation pathways. PLoS One. 2013;8(4):e61934.

21. Huang Y, Sramkoski RM, Jacobberger JW. The kinetics of G2 and M transitions regulated by B cyclins. PLoS One. 2013;8(12):e80861.

22. Loh SF, Cooper C, Selinger CI, et al. Cell cycle marker expression in benign and malignant intraductal papillary lesions of the breast. J Clin Pathol. 2015;68(3):187-191.

23. Fidler IJ. The pathogenesis of cancer metastasis: the 'seed and soil' hypothesis revisited. Nat Rev Cancer. 2003;3(6):453-458.

24. Huber MA, Azoitei N, Baumann B, et al. NF-kappaB is essential for epithelial-mesenchymal transition and metastasis in a model of breast cancer progression. J Clin Invest. 2004;114(4):569-581.

25. Zhang M, Dai C, Zhu H, et al. Cyclophilin A promotes human hepatocellular carcinoma cell metastasis via regulation of MMP3 and MMP9. Mol Cell Biochem. 2011;357(1-2):387-395.

26. Staack A, Badendieck S, Schnorr D, et al. Combined determination of plasma MMP2, MMP9, and TIMP1 improves the non-invasive detection of transitional cell carcinoma of the bladder. BMC Urol. 2006;6:19.
Drug Design, Development and Therapy

\section{Publish your work in this journal}

Drug Design, Development and Therapy is an international, peerreviewed open-access journal that spans the spectrum of drug design and development through to clinical applications. Clinical outcomes, patient safety, and programs for the development and effective, safe, and sustained use of medicines are a feature of the journal, which

\section{Dovepress}

has also been accepted for indexing on PubMed Central. The manuscript management system is completely online and includes a very quick and fair peer-review system, which is all easy to use. Visit http://www.dovepress.com/testimonials.php to read real quotes from published authors.

Submit your manuscript here: http://www.dovepress.com/drug-design-development-and-therapy-journal 